\title{
Toward the Use of NanoSQUIDs to Measure the Displacement of an NEMS Resonator
}

\author{
Trupti Patel, Bo Li, Tianyi Li, Rui Wang, John C. Gallop, David C. Cox, Jie Chen, Edward J. Romans, and Ling Hao
}

\begin{abstract}
We discuss steps toward the readout of the position of a nanoelectromagnetic system (NEMS) beam resonator using a $\mathrm{Nb}$ nanosuperconducting quantum interference device (nanoSQUID). We describe our fabrication procedure for coupling the nanoSQUID and a suspended Al-coated $\mathrm{Si}_{3} \mathrm{~N}_{4}$ NEMS resonator together by a combination of focused ion beam lithography and nanomanipulation. We discuss typical electrical characteristics of the integrated devices, and independent postfabrication atomic force microscope nanoindentation measurements of the elastic properties of the integrated resonator to estimate its natural frequencies of vibration. We compare and discuss the response of a nanoSQUID with current-carrying and superconducting screening (noncurrent carrying) modes of operation of the resonator.
\end{abstract}

Index Terms-NanoSQUIDs, SQUIDs, nanoelectromechanical systems, NEMS, flux coupling, nanomechanical resonator.

\section{INTRODUCTION}

$\mathbf{S}$ QUIDS (Superconducting Quantum Interference Devices) are ultrasensitive detectors of magnetic flux and through suitable input transduction may be used to measure a wide range of physical parameters including electrical currents, photon energies, or displacements of mechanical sensors [1]. NanoSQUIDs are increasingly being developed as they offer high flux and spin sensitivity due to their reduction in size [2][4]. They have direct applications in scanning SQUID systems [5], transition edge sensors [6] and quantum computation [7].

The growing interest in nanoelectromechanical systems (NEMS) devices is due to the vast array of potential applications

Manuscript received September 6, 2016; accepted February 7, 2017. Date of publication February 17, 2017; date of current version February 24, 2017. This work was supported in part by the U.K. National Measurement System Programme, in part by the European Metrology Research Programme project "MetNEMS," in part by the U.K. Engineering and Physical Sciences Research Council, and in part by the U.K. National Institute for Health Research. (All authors contributed equally to this work.) (Corresponding author: Trupti Patel.)

T. Patel is with the London Centre for Nanotechnology, University College London, London WC1H 0AH, U.K., and also with the National Physical Laboratory, Teddington TW11 0LW, U.K. (e-mail: t.patel.12@ucl.ac.uk).

B. Li, R. Wang, J. C. Gallop, D. C. Cox, and L. Hao are with the National Physical Laboratory, Teddington TW11 0LW, U.K. (e-mail: bo.li@ npl.co.uk; rui.wang@npl.co.uk; john.gallop@npl.co.uk; david.cox@npl.co.uk; ling.hao@npl.co.uk).

T. Li and E. J. Romans are with the London Centre for Nanotechnology, University College London, London WC1H 0AH, U.K. (e-mail: tianyi. li.14@ucl.ac.uk; e.romans@ucl.ac.uk).

J. Chen is with the School of Engineering and Design, Brunel University, Uxbridge UB8 3PH, U.K. (e-mail: jie.chen@ brunel.ac.uk).

Color versions of one or more of the figures in this paper are available online at http://ieeexplore.ieee.org.

Digital Object Identifier 10.1109/TASC.2017.2667404 in both quantum and mechanical measurements [8]. At low temperatures, nanoscale mechanical resonators have already been shown to exhibit quantum behavior [9]. By coupling the resonator with a SQUID we hope to convert ultra-small displacements in the position of a NEMS resonator into an electrical readout given by a SQUID.

We have chosen to develop nanoscale SQUIDs (nanoSQUIDs) as readouts due to their high intrinsic flux sensitivity [10] resulting from their low geometric inductance, $L$, and also because we would like to match the scale of the SQUID to the resonator. The latter consideration allows us to firstly optimize the sensing area of the SQUID loop so we can measure the displacement directly with no input transduction, and secondly to permit easier fabrication processes which for instance may be partially done in-situ. In order to couple signals to conventional microscale SQUIDs, generally input coils or flux transformers are used. However with nanoscale SQUIDs, the fabrication and integration of multi-turn input coils becomes difficult, so the preferred method is to directly measure the displacement of the NEMS resonator using the nanoSQUID.

In previous work we have reported on the development of nanoSQUIDs coupled to paddle or beam shaped Al- or Aucoated Si resonators [11]-[13]. In the present work we instead report on devices coupled to Al-coated $\mathrm{Si}_{3} \mathrm{~N}_{4}$ beam shaped resonators. The use of $\mathrm{Si}_{3} \mathrm{~N}_{4}$ with its higher Young's modulus, and the low mass of the beam configuration allows potentially higher resonant frequencies, which should lead to greater sensitivity in mass or chemical detection applications.

\section{THE FABRICATION PROCESS}

\section{A. Resonator}

The resonating beam was fabricated from aluminum coated silicon nitride $\left(\mathrm{Si}_{3} \mathrm{~N}_{4}\right)$. The aluminum layer was $40 \mathrm{~nm}$ thick and the silicon nitride was $100 \mathrm{~nm}$ thick. $\mathrm{Si}_{3} \mathrm{~N}_{4}$ was used as group III nitrides have exhibited high structural quality and dimensional control down to a monolayer [14]. In addition they exhibit piezoelectricity which could provide additional future functionality to the device. An alternative resonator material would be aluminum nitride which has already achieved quality factors in the tens of thousands [14]. The $\mathrm{Si}_{3} \mathrm{~N}_{4}$ films were grown on silicon substrates and were patterned using optical lithography. These were then undercut by a two stage etching 


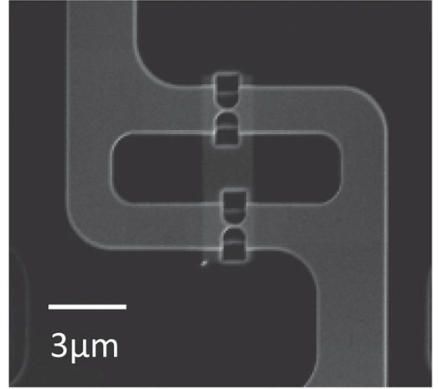

Fig. 1. SEM image of a typical $\mathrm{Nb}$ nanoSQUID before the integration of the resonator structure on top. The SQUID loop and connecting tracks were fabricated by optical lithography. The two nanobridges were fabricated by FIB and are approximately $60 \mathrm{~nm}$ wide.

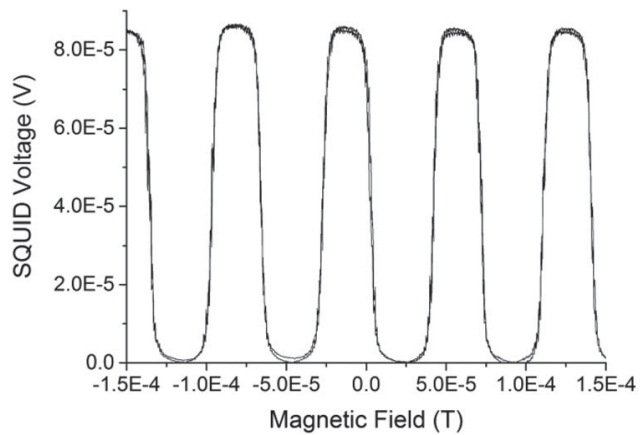

Fig. 2. Voltage modulation curve of a typical $\mathrm{Nb}$ nanoSQUID at $7.8 \mathrm{~K}$. The asymmetry in the curve is due to the Earth's magnetic field.

process producing double clamped beams of varying lengths $(20-50 \mu \mathrm{m})$ and widths $(2-5 \mu \mathrm{m})$.

\section{B. $S Q U I D$}

The SQUIDs used had loop dimensions of $3 \mu \mathrm{m} \times 10 \mu \mathrm{m}$ designed to match the scale of the resonators as shown in Fig. 1. For ultimate sensitivity it would be desirable in future to further reduce the dimensions of both the resonator and SQUID. However at this stage we chose to work with slightly larger resonators that could be easily manipulated and pre-stressed during attachment in the FIB system. This ensured they had a curved profile and would not come into contact with the SQUID. The SQUIDs were fabricated from an optically pre-patterned $160 \mathrm{~nm}$ thick niobium film using an FEI Nova NanoLab 600 dual-beam Focused Ion Beam (FIB). Dayem-type nanobridge weak links of approximately $60 \mathrm{~nm}$ in length and width were fabricated within the niobium pre-patterned loop to act as the Josephson elements [15]. The nanobridge regions were coated with amorphous e-beam deposited tungsten before the milling process. There are two benefits due to this. Firstly the device is partially protected from implantation poisoning which may occur during the milling process. Secondly it provides the nanobridge region with a normal thermal shunt layer allowing it to operate over a wider range of temperatures without hysteretic $I V$-characterstics due to hotspot formation.

A typical voltage-field of the nanoSQUIDs is shown in Fig. 2 corresponding to a voltage-flux transfer function, $V_{\Phi} \equiv$ $(\partial V / \partial \Phi)_{\max }=560 \mu \mathrm{V} / \Phi_{0}$ at $7.8 \mathrm{~K}$. Previous measurements

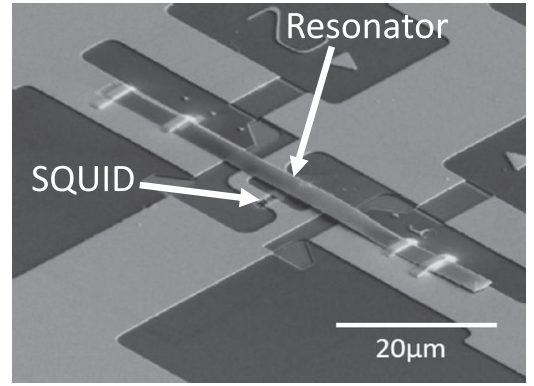

Fig. 3. SEM image of a fully integrated device. A $3 \mu \mathrm{m} \times 30 \mu \mathrm{m}$ Al-coated $\mathrm{Si}_{3} \mathrm{~N}_{4}$ beam resonator is suspended above the long side of a $3 \mu \mathrm{m} \times 10 \mu \mathrm{m}$ SQUID loop. The Nb SQUID loop and connecting tracks were fabricated by FIB lithography from an optically pre-patterned $\mathrm{Nb}$ thin film. (The connecting tracks to the SQUID and resonator were originally shorted together in the pre-patterned design and later cut using the FIB as can be seen in the image.).

of the SQUID noise of similar devices at $7.8 \mathrm{~K}$ gave a very low noise floor of around $0.2 \mu \Phi_{0} \mathrm{~Hz}^{1 / 2}$ at $1 \mathrm{kHz}$.

\section{Coupled Devices}

The Al-coated $\mathrm{Si}_{3} \mathrm{~N}_{4}$ resonators for all devices were attached to the SQUID in the FEI Nova NanoLab 600 in-situ using a piezo-based nano-manipulation system. The manipulator is connected to the resonator situated on a separate chip adjacent to the chip on which the SQUID is located. The manipulator is attached by ion beam deposited platinum $(\mathrm{Pt})$ and is cut from the substrate once attached to the tip using the gallium ion beam. It is then moved into close proximity to the SQUID. As the sample stage cannot be rotated with respect to the tip some care is required in aligning the beam and the SQUID chips such that they still align once the resonator is cut from the initial chip and moved to the SQUID device. The final stage involves the deposition of a small amount of Pt at the ends of the beam to ensure an electrical connection between the aluminum film on the $\mathrm{Si}_{3} \mathrm{~N}_{4}$ beam and the pre-patterned contact leads on the SQUID chip. A typical completed device is shown in Fig. 3. The resonator is asymmetrically positioned over one side of the SQUID loop in order to maximise the response from the SQUID. If the resonator had been aligned along the centre axis of the SQUID loop, a current flowing along the beam would produce no net flux in the SQUID.

\section{PRedicted Resonant Frequency Using AFM}

\section{A. Nanoindentation}

One problem we have previously found with other types of resonators coupled to SQUIDs [12] is that there is often a discrepancy between the ideal theoretical and the measured resonant frequencies which is likely due to non-ideal materials and imperfections in fabrication. It is therefore highly desirable to be able to characterize the elastic properties of coupled resonators independently of any SQUID measurement. To determine the spring constant of the resonator and the Young's modulus of the material $E$ in the final integrated device, we used the technique of nanoindentation. This has been used by other groups to characterize nanowires [16] and uses an Atomic Force Microscope 


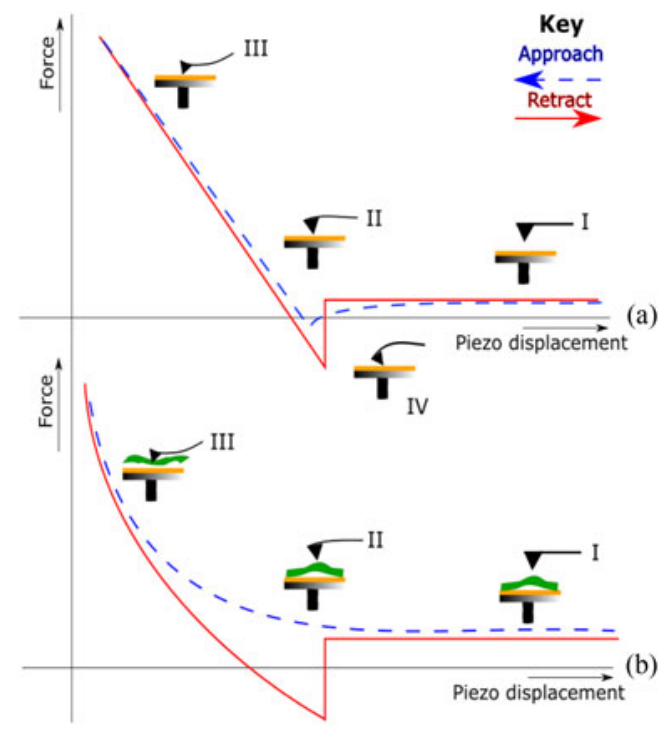

Fig. 4. Principle of nanoindentation. As the tip approaches the surface of the sample from afar in region I, the AFM cantilever is undeflected. At II the tip is attracted to the sample surface via the van der Waals force. In region II to III the cantilever is bent as the tip presses into the sample. From III to IV the cantilever bends in the opposite direction due to adhesion forces as it attempts to retract from the sample. The differences between the approach and retract curves are due to contact hysteresis. (a) shows the case of a hard structure and (b) shows the case for the resonator, a flexible structure.

(AFM) to precisely measure the interaction forces between the tip and the resonator. During the process of nanoindentation, the AFM cantilever tip approaches and is pushed into the sample until a predefined maximum force is exerted onto the sample; once this value has been reached the tip is retracted. During the procedure both the AFM piezo displacement and the force exerted onto the sample are recorded as shown in Fig. 4, with the spring constant then extracted from the slope. Since the sample also bends, what is actually measured is the total spring constant $k_{\Sigma}$ for the combined cantilever and resonator. This satisfies

$$
1 / k_{\sigma}=1 / k_{R}+1 / k_{C}
$$

where $k_{\mathrm{R}}$ is the spring constant of the resonator alone and $k_{\mathrm{C}}$ is the spring constant of the cantilever alone. We determined the latter separately through the method of thermal tuning [12]. Using (1) and the measured values of $k_{\mathrm{C}}$ and $k_{\Sigma}$, we then estimated the effective value of $k_{\mathrm{R}}$ against AFM tip position along the beam as shown in Fig. 5 .

As would be expected the effective spring constant is lower in the center of the resonator. For a doubly-clamped beam of length $l$, width $w$, thickness $t$ and rectangular cross-section loaded at distance $a$ from one end, the standard expression for the effective spring constant at the load point is $k=3 E I l^{3} / a^{3}(l-a)^{3}$, where $I=w t^{3} / 12$ is the area moment of inertia. A fit to this is shown in Fig. 5 for $w=3 \mu \mathrm{m}, t=140 \mathrm{~nm}, l=30 \mu \mathrm{m}$ giving a best fit value of $E=225 \mathrm{GPa}$. We can compare this with a theoretical estimate using typical ideal values of the Young's modulus for the bilayer $\left(E_{\mathrm{Al}}=70 \mathrm{GPa}, E_{\mathrm{SiN}}=310 \mathrm{GPa}\right)$ and thicknesses $\left(t_{\mathrm{Al}}=40 \mathrm{~nm}, t_{\mathrm{SiN}}=100 \mathrm{~nm}\right)$. This gives $E=\left(t_{\mathrm{SiN}} E_{\mathrm{SiN}}+t_{\mathrm{Al}} E_{\mathrm{Al}}\right)\left(t_{\mathrm{SiN}}+t_{\mathrm{Al}}=241 \mathrm{GPa}\right.$. The experimental value is in reasonably good agreement despite the

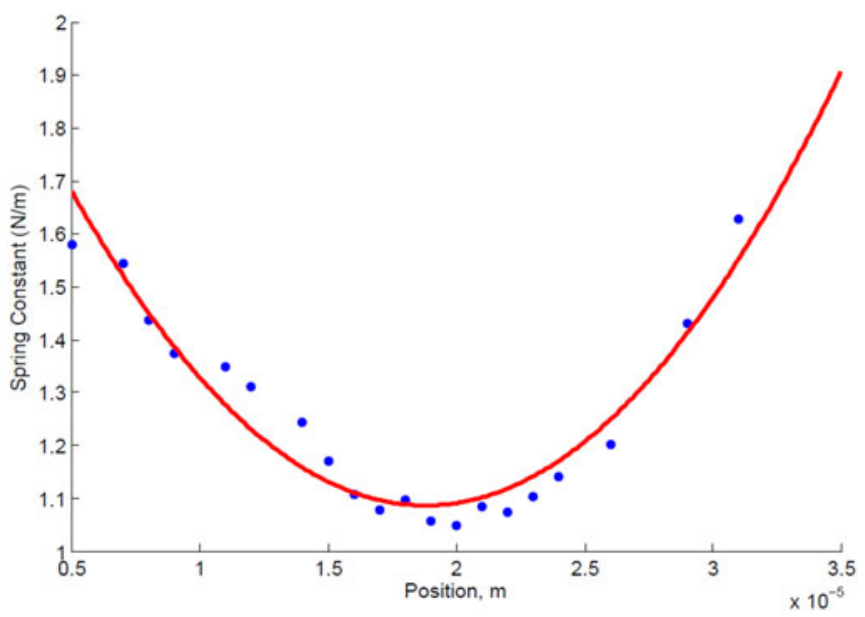

Fig. 5. Effective spring constant $k_{\mathrm{R}}$ of the $\mathrm{Si}_{3} \mathrm{~N}_{4}$ beam resonator determined versus position along a $30 \mu \mathrm{m}$-long beam. (The extent of the horizontal axis shown corresponds to the suspended part the beam.) The line is a best fit to the theory with Young's modulus $E=225 \mathrm{GPa}$.

slightly arched beam profile, possible additional tension caused by the deposited Pt contacts, and non-ideal material quality.

From this we can make a reasonable estimate of the frequencies of the natural modes of vibration of the resonator which can be determined by Fourier analysis of the solutions of the Euler-Lagrange equation with appropriate boundary conditions. For a doubly-clamped uniform beam, the natural frequency $f_{1}$ of the lowest mode is predicted [18] to be

$$
f_{1}=\frac{3.56}{l^{2}} \sqrt{\frac{E I}{\rho A}}
$$

where $l$ is the length, $\rho$ is the density, and $A$ is the cross-sectional area. Using the measured value of $E$ and textbook values of $3200 \mathrm{kgm}^{-3}$ and $2700 \mathrm{kgm}^{-3}$ for the respective densities of $\mathrm{Si}_{3} \mathrm{~N}_{4}$ and $\mathrm{Al}$, we estimate the first mode frequency ranges from $0.49 \mathrm{MHz}$ for a $50 \mu \mathrm{m}$-long beam to $3.09 \mathrm{MHz}$ for a $20 \mu \mathrm{m}$-long beam with $w=3 \mu \mathrm{m}$ and $t=140 \mathrm{~nm}$.

\section{Measuring the Resonator Using the SQUID}

\section{A. Comparison of a Conducting and Superconducting Resonator}

For NEMS readout we use the nanoSQUID in small signal mode. The nanoSQUID is current biased above its critical current, with a perpendicular dc magnetic field $B_{\mathrm{SQU}} \mathrm{UI}=$ $4.2 \times 10^{-5} \mathrm{~T}$ applied to flux bias the SQUID onto its working point. Although the SQUIDs have steep $V$ - $\Phi$ characteristics, we have found in previous work that there is no issue with stability at the working point in small signal mode. Since the resonator in this case is Al-coated there are in principle two ways it could be used with an integrated SQUID readout. The first is to use the resonator as a current carrying conductor so that the motion of the resonator simply changes the flux detected by the SQUID. The motion of the resonator gives a time-varying mutual inductance $M(t)=M_{0} \sin \omega t$ between the resonator and the SQUID. Using an ac resonator current $I_{\mathrm{R}}(t)=I_{0} \sin \omega t$, 


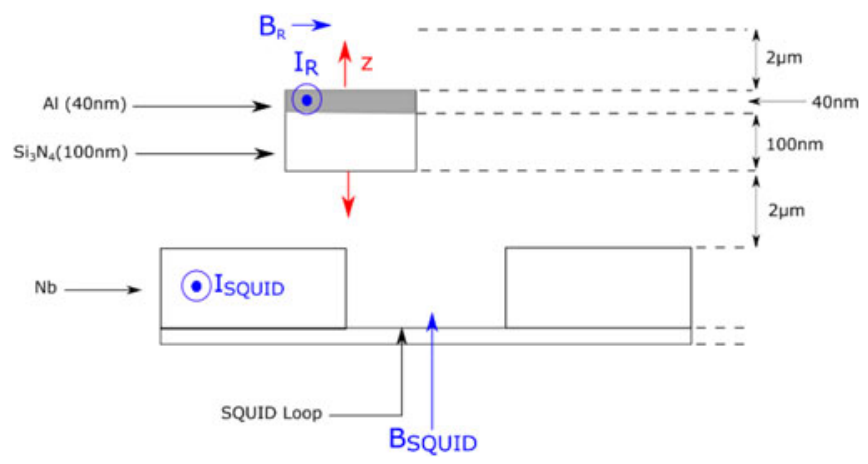

Fig. 6. Cross-sectional view (not to scale) of the resonator above the SQUID loop showing the dimensions used in the inductance modelling and the directions of the applied magnetic fields. The red arrows show the direction in which the resonator vibrates.

combined with an additional in-plane dc magnetic field $B_{\mathrm{R}}$, the beam can be continuously driven via a time-varying Lorentz force $F(t)=B_{\mathrm{R}} I_{\mathrm{R}}(t) l$. The orientation of the two applied magnetic fields is shown in Fig. 6. The flux in the SQUID, $\Phi(t)=M(t) I_{\mathrm{R}}(t)$ would then have a component at twice the excitation frequency which could be detected with e.g. a lock-in amplifier.

The second approach is to use the superconducting coated resonator below its superconducting transition temperature, without an applied current. In this case the motion of the superconducting resonator modulates the self-inductance $L$ of the SQUID changing its output voltage. The field due to the circulating supercurrent in the SQUID generates a screening current in the resonator which also generates its own field. During displacement of the resonator, the interaction between the two fields change as the separation changes. Thus the magnetic field profile of the SQUID is altered. In turn this creates a change in the output voltage of the SQUID given by

$$
\delta V \approx-\left(\frac{\partial V}{\partial \Phi}\right) \frac{\Phi_{\mathrm{ext}}}{L} \frac{d L}{d z} \delta z
$$

where $\Phi_{\text {ext }}$ is the applied flux perpendicular to the SQUID, $d L$ is the change in self-inductance of the SQUID loop due to the displacement of the resonator, and $\delta z$ is the vertical displacement of the resonator from rest.

We used the superconducting inductance package 3D-MLSI to model the relevant mutual and self inductances for the two cases versus the resonator position. The relevant dimensions of the device are indicated on Fig. 6. The neutral position of the resonator is $2 \mu \mathrm{m}$ above the SQUID. In the horizontal direction, zero is taken to be the center of the SQUID loop with the nanobridges (not shown) positioned at $\pm 1.5 \mu \mathrm{m}$. The mutual inductance between the SQUID loop and resonator for various horizontal and vertical offsets of the resonator is shown in Fig. 7. As the resonator moves away from the SQUID loop the mutual inductance decreases as expected. In the fabricated device the resonator was aligned as close as possible over one of the nanobridges to maximize the coupling, so we used this horizontal offset for the subsequent modelling of both cases.

The change in SQUID voltage was then predicted for the two cases for given vertical displacements of the resonator as

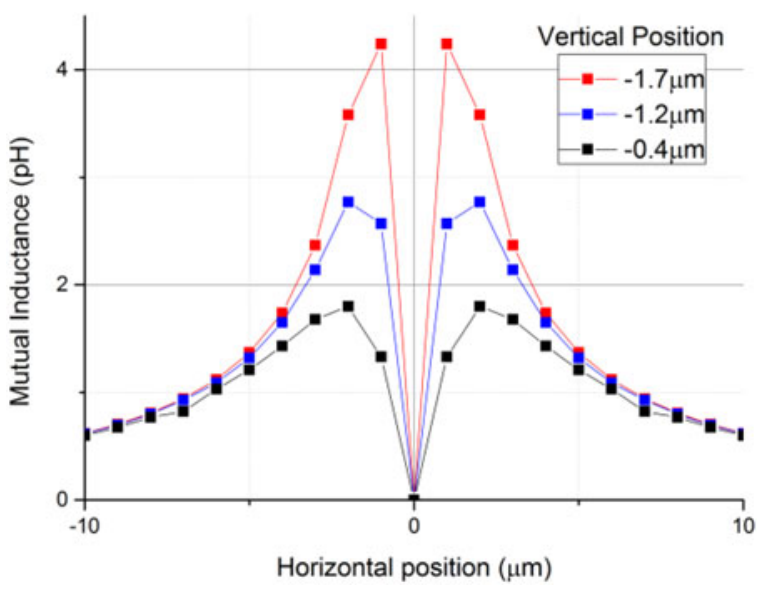

Fig. 7. Mutual inductance between the beam and the nanoSQUID for three representative vertical displacements as a function of the horizontal position of the resonator. The vertical displacement is with respect to the neutral position, $2 \mu \mathrm{m}$ above the SQUID. The mutual inductance is zero when the resonator axis is symmetrically aligned along the centre of the SQUID loop, and maximum when the resonator is aligned above the nanobridges.

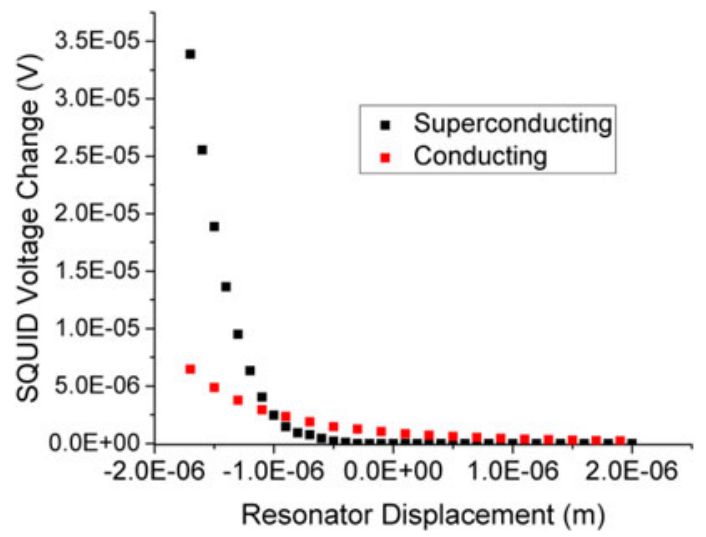

Fig. 8. Predicted SQUID response versus vertical displacement for a superconducting and conducting resonator. In both cases the resonator is aligned above one of the nanobridges with zero displacement corresponding to the neutral position $2 \mu \mathrm{m}$ above the SQUID. The conducting case assumes that the resonator is carrying a current of magnitude $15 \mu \mathrm{A}$

shown Fig. 8. The current-carrying case was modelled for a resonator current of $15 \mu \mathrm{A}$, which keeps the SQUID in small signal mode. For this particular current and beam-to-SQUID separation, the conducting current-carrying resonator gives a slightly larger voltage swing than the superconducting resonator for small displacements, whereas the superconducting resonator gives a larger response when it moves closer to the SQUID loop.

Given the (white) voltage noise floor of the nanoSQUID is $\sim 1 \times 10^{-10} \mathrm{~V} / \mathrm{Hz}^{1 / 2}$, the steepest parts of the responses shown in Fig. 8 correspond to maximum displacement sensitivities of respectively $5.71 \times 10^{-12} \mathrm{~m} / \mathrm{Hz}^{1 / 2}$ and $3.34 \times$ $10^{-11} \mathrm{~m} / \mathrm{Hz}^{1 / 2}$ for the superconducting and conducting resonators.

\section{CONClusion}

We show in this paper the principle behind nanoSQUID readout of a NEMS resonator and the expected response of the 
nanoSQUID when the resonator is used as a current-carrying conductor or as a superconducting shield. The nanoSQUIDNEMS coupled devices may all be made in-situ using a gallium FIB making the fabrication process much simpler. The spring constant of the resonator was measured using the process of nanoindentation allowing an estimation of the natural frequencies. Future work will be undertaken to experimentally detect the motion of the resonator through a SQUID.

Although the SQUID response for a conducting resonator is of a similar order of magnitude and the response may be increased by increasing the current through the resonator, there are several key reasons to pursue superconducting resonators including the lack of dissipation, the removal of the need for direct current connections if another mode of actuation is employed, and the potential for very high quality factors. If quality factors of superconducting resonators superseded their conducting counterparts by several orders of magnitude the output voltage for devices incorporating these materials would be greater. On the other hand the conducting resonator offers a straightforward way to implement actuation and readout at the same time.

One method of actuation currently being considered is magnetic. The advantage of this technique is that it is broadband [19]. If a superconducting resonator was used, magnetic actuation would be complicated by the Meissner effect of the superconductor, so that an alternative might need to be considered. Common alternatives include thermal, electrostatic, and piezoelectric actuation. The limiting factor for thermal actuation is the thermal time constant, the time it takes to cool the device down before it may be heated again [20]. For piezoelectric actuation it is often the charging time of the actuation circuit which limits the frequency of actuation [21], and for electrostatic actuation it is the parasitic capacitance of the chip which may dominate at high frequencies [22]. For superconducting devices, thermal actuation might be a good alternative due to the low thermal time constant at cryogenic temperatures. This could for instance achieved through the use of a laser or an on-chip heating element.

\section{REFERENCES}

[1] The SQUID Handbook, vol. I, J. Clarke and A. I. Braginski, Ed. New York, NY, USA: Wiley, 2006.

[2] C. Granata and A. Vettoliere, "Nano superconducting quantum interference device: A powerful tool for nanoscale Investigations," Phys. Rep., vol. 614, pp. 1-69 2016.
[3] C. Foley and H. Hilgenkamp, "Why NanoSQUIDs are important: An introduction to the focus issue," Supercond. Sci. Technol., vol. 22, 2009, Art. no. 064001 .

[4] J. Gallop, P. W. Joseph-Franks, J. Davies, L. Hao, and J. Macfarlane, "Miniature dc SQUID devices for the detection of single atomic spin-flips," Physica C, vol. 368, pp. 109-113, 2002.

[5] A. Finkler et al., "Scanning superconducting quantum interference device on a tip for magnetic imaging of nanoscale phenomena," Rev. Sci. Instrum., vol. 83, 2012, Art. no. 073702.

[6] L. Hao et al., "Inductive superconducting transition-edge detector for single-photon and macro-molecule detection," Supercond. Sci. Technol., vol. 16, pp. 1479-1482, 2003.

[7] J. Tejeda et al., "Magnetic qubits as hardware for quantum computation," Nanotechnology, vol. 12, pp. 181-186, 2001.

[8] K. L. Ekinci and M. L. Roukes, "Nanoelectromechanical systems," Rev Sci. Instrum., vol. 76, 2005, Art. no. 061101.

[9] J. D. Teufel et al., "Sideband cooling of micromechanical motion to the quantum ground state," Nature, vol. 475, pp. 359-363, 2011.

[10] T. Patel et al., "Investigating the intrinsic noise limit of nanoSQUIDs," IEEE Trans. Appl. Supercond., vol. 25, no. 3, Jun. 2015, Art. no. 1602105.

[11] L. Hao et al., "Focussed ion beam nanoSQUIDs as novel NEMS resonator readout," IEEE Trans. Appl. Supercond., vol. 19, no. 3, pp. 693-696, Jun. 2009.

[12] L. Hao et al., "Coupled nanoSQUIDs and nano-electromechanical systems (NEMS) resonators," IEEE Trans. Appl. Supercond., vol. 23, no. 3, Jun. 2013, Art. no. 1800304.

[13] S. Bechstein et al., "Design and fabrication of coupled nanoSQUIDs and NEMS," IEEE Trans. Appl. Supercond., vol. 25, no. 3, Jun. 2015, Art. no. 1602604.

[14] A. N. Cleland, M. Pophristic, and I. Ferguson, "Single-crystal aluminum nitride nanomechanical resonators," Appl. Phys. Lett., vol. 79, 2001, Art. no. 2070.

[15] L. Hao, D. Cox, and J. Gallop, "Characteristics of focused ion beam nanoscale Josephson devices," Supercond. Sci. Technol., vol. 22, 2009, Art. no. 064011 .

[16] A. San Paulo et al., "Mechanical elasticity of single and double clamped silicon nanobeams fabricated by the vaporliquid-solid method," Appl. Phys. Lett., vol. 87, 2005, Art. no. 053111 .

[17] J. L. Hutter and J. Bechhoefer, "Calibration of atomic-force microscope tips," Rev. Sci. Instrum., vol. 64, pp. 1868-1873, 1993.

[18] R. D. Blevins, Formulas for Natural Frequency and Mode Shape. New York, NY, USA: Van Nostrand Reinhold, 1979.

[19] X. M. H. Huang, C. A. Zorman, M. Mehregany, and M. L. Roukes, "Nanoelectromechanical Systems: Nanodevice motion at microwavexbrk frequencies," Nature, vol. 421, p. 496, 2003.

[20] B. Ilic, S. Krylov, K. Aubin, R. Reichenbach, and H. G. Craighead, "Optical excitation of nanoelectromechanical oscillators," Appl. Phys. Lett., vol. 86, 2005, Art. no. 193114.

[21] M. Li, H. X. Tang, and M. L. Roukes, "Ultra-sensitive NEMS-based cantilevers for sensing, scanned probe and very high-frequency applications," Nature Nanotechnol., vol. 2, pp. 114-120, 2007.

[22] L. Sekaric, J. M. Parpia, H. G. Craighead, T. Feygelson, B. H. Houston, and J. E. Butler, "Nanomechanical resonant structures in nanocrystalline diamond," Appl. Phys. Lett., vol. 81, pp. 4455-4457, 2002. 